\title{
Differential use of distance and location information for spatial localization
}

\author{
RICHARD A. ABRAMS and JONATHAN Z. LANDGRAF \\ Washington University, St. Louis, Missouri
}

\begin{abstract}
Five experiments are reported in which subjects judged the movement or spatial location of a visible object that underwent a combination of real and induced (illusory) motion. When subjects attempted to reproduce the distance that the object moved by moving their unseen hands, they were more affected by the illusion than when they pointed to the object's perceived final location. Furthermore, pointing to the final location was more affected by the illusion when the hand movement began from the same position as that at which the object initially appeared, as compared with responses that began from other positions. The results suggest that people may separately encode two distinct types of spatial information: (1) information about the distance moved by an object and (2) information about the absolute spatial location of the object. Information about distance is more susceptible to the influence of an induced motion illusion, and people appear to rely differentially on the different types of spatial information, depending on features of the pointing response. The results have important implications for the mechanisms that underlie spatially oriented behavior in general.
\end{abstract}

The human perceptual system has the remarkable ability to maintain a stable perception of the egocentric directions (i.e., directions relative to the head) of objects in the world, despite the fact that the portion of the retina stimulated by an object changes as the eyes move. This phenomenon, known as visual direction constancy, allows people to move about and interact, often quite gracefully, with objects in their environment.

Although the visual direction of moving and stationary objects is usually perceived accurately, systematic departures from accurate localization have been demonstrated in a number of situations (e.g., Festinger \& Canon, 1965; Hill, 1972; Matin, Matin, \& Pearce, 1969; Morgan, 1978). The conditions that induce mislocalization have been the subject of a substantial amount of research since the time of Helmholtz (1866/1963), because it is believed that an understanding of the failures of visual direction constancy can lead to a better understanding of the mechanisms that underlie accurate perceptual-motor behavior. The present article continues in that spirit.

Two interrelated but distinct issues are central to the study of spatial localization. The first involves the way in which different patterns of stimulation may alter the information available to an observer about the location of an object. The second issue involves the extent to which differences in the responses that people must produce toward some object reflect differences in the available spa-

This research was supported by Biomedical Research Support Grants BRSG S07 RR07054-22 and -23 from the National Institutes of Health, and a Washington University Faculty Research Grant. The authors thank Myron Braunstein, Arien Mack, and an anonymous reviewer for helpful comments on an earlier draft of the manuscript, and Adam Kahan for technical assistance. Correspondence concerning this article should be sent to Richard A. Abrams, Department of Psychology, Washington University, St. Louis, MO 63130. tial information. Some researchers have chosen to explore the former issue, whereas others have focused on the latter. For example, a number of researchers have examined localization as a function of the number and type of eye movements that subjects use to fixate a target to be localized (Festinger \& Canon, 1965; Honda, 1984, 1985), or as a function of the retinal location of the target (Hill, 1972; Morgan, 1978). These studies have mainly been concerned with potential differences in the information available about a visual target after different types of stimulation. Others have compared localization using aimed eye or limb movements as a response, with localization that required subjects to make perceptual judgments (e.g., Bridgeman, Lewis, Heit, \& Nagle, 1979). The goal in these studies has been to learn about the nature of the spatial information that was available to different output systems.

The approaches described above have yielded considerable insight into the nature of the mechanisms underlying spatial localization. However, they each have a potential limitation due to the fact that they focus primarily on features of only the visual stimulus, or on manipulations of only the required localization responses. Such approaches may obscure important interactions between the spatial information available about an object, and the use of that information to produce appropriate responses. For example, inaccurate spatial localization in a given situation could arise from inadequate encoding of the information in the stimulus, or from inaccessibility of the information for use in a particular response, or both. If one manipulates only stimulus factors, or only response factors, it can be difficult to make general conclusions about localization mechanisms. In the present paper, we study manipulations of both the visual stimulus that subjects are asked to localize, and the nature of the response that they are asked to produce. Our goal is to learn more about both 
the perceptual and the motor mechanisms underlying spatial localization of seen objects.

\section{Localization by Perceptual and Motor Systems}

Perhaps one of the most interesting results in the localization literature is the fact that the fidelity of the spatial information available about a seen object depends heavily on the nature of the localization response that subjects must produce. In a number of studies, subjects have been asked to locate a visual stimulus that was briefly flashed in an otherwise dark environment during or near the time of an eye movement. When subjects are asked to provide perceptual judgments of the position of the flash (e.g., by moving a light to its perceived location, or by judging its position relative to a previously seen reference point) considerable errors are observed (Matin, 1972; Matin et al., 1969). However, when asked to point their eyes or a limb to the location of the flash (a motor response), they are able to respond quite accurately (Hallett \& Lightstone, 1976; Hansen, 1979; Hansen \& Skavenski, 1977, 1985; Miller, 1980). Thus, the motor system appears to have access to spatial information that is unavailable to the perceptual system.

The superiority of localization by means of the motor system has also been shown in several other situations. People are able to accurately point an unseen hand in the direction of a visual target that has been displaced slightly (motor response), even though they are unaware that a displacement has occurred (perceptual response; Bridgeman, Kirch, \& Sperling, 1981; Bridgeman et al., 1979). And people can accurately direct their eyes to a stationary visual stimulus, despite the fact that they perceive that the stimulus has moved (Wong \& Mack, 1981) or is moving (Lackner \& Levine, 1981).

These results have important implications for the processes involved in extracting information from some internal "map" of visual space. Apparently, different brain systems that might be involved in locating a visible object do not all have equal access to that spatial map. Rather, the information available to the motor systems for controlling eye or hand movement appears to be more reliable than that available to the perceptual/cognitive system.

One can readily appreciate the adaptive significance of the motor system's relative immunity to factors that affect perceptual judgments of spatial location. There are, however, reasons to question the generality of this conclusion. In a number of situations, motoric orienting responses (such as pointing the eye or an unseen limb) have been shown to be affected by the same spatial illusions and distortions that affect perceptually based localization (saccadic vs. smooth eye movements: Abrams, Meyer, \& Kornblum, in press; Festinger \& Canon, 1965; Honda, 1985; Miller, 1980; induced motion illusion: Bacon, Gordon, \& Schulman, 1982; Bridgeman et al., 1981; Mack, Heuer, Fendrich, Villardi, \& Chambers, 1985; Wong \& Mack, 1981; Müller-Lyer illusion: Coren, 1986; Mack, Heuer, Villardi, \& Chambers, 1985; global effect: Findlay, 1982; oculoparalytic illusion: Matin et al., 1982; proprioceptive illusions: Lackner \& Levine, 1981). Evidently, the motor system sometimes has access to spatial information that is unaffected by various distortions, whereas at other times, either it does not have access to that information, or that information is distorted.

\section{A New Theoretical Perspective}

The inconsistent differences in localization accuracy that have been found when perceptual and motor responses are compared present something of a puzzle. On the basis of the existing evidence, it would be difficult to predict the circumstances under which the motor system might have access to information that is unavailable to the perceptual system. Furthermore, it is not clear precisely what the nature of the information might be that is sometimes available to the motor system but not to the perceptual system. We hope to provide at least a tentative solution to this puzzle by viewing localization from a perspective that is somewhat different from that taken in the past.

Our premise is simple: It is theoretically possible that there is more than one type of spatial information available for localization. Different types of stimuli may selectively influence a person's ability to encode the different types of information, and different localization responses may depend differentially on the various types of information. Our goal in the present article is to provide general support for these possibilities.

Dissociating distance and location information. In the present experiments, we explored the possibility that there exist two specific types of spatial information: (1) information about the distance between an object and some reference object or position, and (2) information about the absolute location of an object. Of course, in Euclidean coordinate systems, distances and locations are always linked together. Nevertheless, there is reason to believe that people may perceive each of these spatial attributes "directly" (i.e., one is not derived from the other). For example, Mack, Heuer, Villardi, and Chambers (1985) have shown that the perceived distance between the shaftends in a Müller-Lyer figure (i.e., the perceived length of the shaft) does not completely correspond to differences in the perceived locations of the endpoints.

Motor short-term memory. Additional support for a distinction between distance and location information comes from research on the memory for previously produced limb movements. When subjects are asked to reproduce features of a previous movement, they are often better able to reproduce the end location than they are to reproduce the distance of the movement from a new starting position (Kelso, 1977; Marteniuk \& Roy, 1972; Stelmach, Kelso, \& Wallace, 1975). In addition, memory for the distance and the end location of a previously made movement are subject to different interference and decay effects (Laabs, 1973). Thus the two kinds of information appear to be different.

Rapid aimed movements. Considerable support for a distinction between distance and location information is also provided by research on the motor mechanisms in- 
volved in the control of rapid eye and limb movements. Some researchers have characterized such movements as consisting of a preprogrammed burst of activity in agonist muscles with well-defined force-time relationships (for saccadic eye movements: Abrams, Meyer, \& Kornblum, 1989; Robinson, 1964; for limb movements: Abrams et al., in press; Meyer, Abrams, Kornblum, Wright, \& Smith, 1988; Meyer, Smith, \& Wright, 1982; Schmidt, Zelaznik, Hawkins, Frank, \& Quinn, 1979). According to these formulations, the amplitude and duration of the pulse of force is programmed from the outset for a particular movement distance.

Other workers have emphasized the position-seeking aspects of eye and limb movements (as well as movements of the lips and tongue associated with speech). It appears that, in many situations, commands to the muscles may directly specify the final desired end location of a movement (Abrams et al., in press; Bizzi, Polit, \& Morasso, 1976; Kelso, 1977; MacNeilage, 1970; Mays \& Sparks, 1980; Polit \& Bizzi, 1979). In this way, movements can be terminated accurately, regardless of their starting position, and despite unexpected (and undetected) perturbations of the moving member along the way. One way that this may be done is to adjust the desired resting lengths of opposing muscle pairs around the joint that is moving (Polit \& Bizzi, 1979; Sakitt, 1980). Thus, movements are sometimes programmed to arrive at a particular location in space. These ideas are not necessarily inconsistent with the programming of movement distance: Measurements of electromyographic activity in the muscles suggest that both distance and location may be specified in the motor commands (Ghez, 1979; Hallett, Shahani, \& Young, 1975; cf. Abrams et al., in press).

\section{Overview of Present Experiments}

In the present experiments, we explored the possibility that a distinction between spatial information about (1) the distance between an object and some reference position, and (2) the absolute location of an object may provide new insights into details of the mechanisms underlying spatial localization.

To test this possibility, it was necessary to select a visual stimulus that was likely to selectively affect distance and location information. A variation of an induced motion illusion was selected. Induced motion occurs when the movement of a visual object (e.g., a rectangular frame) affects the perceived motion of another object (e.g., a point enclosed within the frame; Duncker, 1929). The illusion is often quite powerful (e.g., see Bacon et al., 1982; Mack, Heuer, Fendrich, et al., 1985), and it has been shown to affect motor-oriented localization responses less than perceptually based responses (Bridgeman et al., 1981). Furthermore, in at least some situations, induced motion produces an illusion of movement without a corresponding change in perceived location (Bacon et al., 1982). Thus, induced motion seemed a good candidate for the possible dissociation of different kinds of spatial information.
In Experiments 1-3, we tested the possibility that perceived distance and location may be different, and that they are differentially affected by induced motion illusions. In Experiment 4, we examined one possible source of the difference between perceived distance and perceived location. In Experiment 5, we explored the possibility that people may implicitly rely differentially on either distance or location information, depending on subtle, often overlooked, features of the localization response.

\section{EXPERIMENT 1}

In most of the previous research directly comparing distance and location information, subjects have been required to recall from memory specific features of a previous limb movement (e.g., Stelmach et al., 1975). In the present experiment, we sought to determine whether differences in spatial information about distance and location also exist when subjects are required to reproduce aspects of the motion of a visual stimulus.

Subjects viewed a visual target that underwent a combination of real and induced motion. They then produced hand movements intended to match either the perceived distance that the target moved, or the perceived final location of the target. If spatial information about distance and location are distinct, and if the induced motion affects one type of information more than the other, then the effect that the illusion has on the responses should depend on whether subjects are judging the final location of the target or the distance through which the target moves.

\section{Method}

Subjects. Eight Washington University undergraduates participated in two 1-h sessions on separate days. None had any apparent visual or motor abnormalities. The subjects were paid a base rate of $\$ 3.50$ per session, plus additional bonus payments based on their performance, and were tested individually.

Apparatus. An IBM PC AT computer controlled the presentation of stimuli and the acquisition of data throughout the experiment. The subjects were seated at a table in a dark room with their eyes $38 \mathrm{~cm}$ from a cathode-ray tube (CRT) display. Their heads were steadied by means of a padded chinrest. The subjects rested their right elbows on a pad on the table, and in their right hands grasped a handle that was mounted on a track along the bottom edge of the CRT. The handle, which was used for making pointing responses, could be moved easily and smoothly from side to side. Its position was monitored by means of a precision potentiometer attached to the track, which sent a position-dependent voltage to the computer. The subjects gripped the handle in their right palms, with the thumb resting on the top of the handle pointing toward the CRT. They understood that an imaginary line extending along the thumb was to be used for pointing to positions on the screen. The subjects pressed a button on the handle to signal that they were satisfied with their response, and the computer recorded the position of the handle at that moment. Even though all pointing was performed in complete darkness, the handle and the subjects' right hands were shielded from view by a piece of cardboard.

Procedure. Figure 1 shows the display that appeared on the CRT at the beginning of each trial. The display included a target (small dot) located directly in front of the subject, surrounded by a rectangular frame $\left(18^{\circ}\right.$ of visual angle wide and $10^{\circ}$ high, centered $4.5^{\circ}$ to the right of the target). At the beginning of each trial, the 


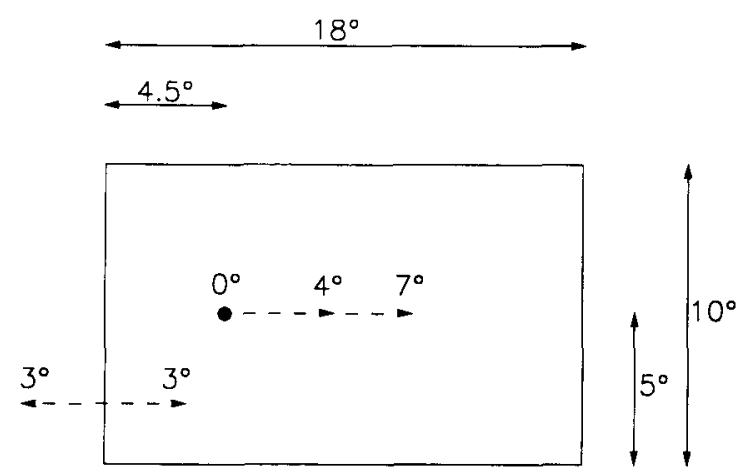

Figure 1. Stimulus display used in Experiment 1. The target (dot) was directly in front of the subject. On each trial, the target moved either $4^{\circ}$ or $7^{\circ}$ to the right, and the frame moved $3^{\circ}$ to the right or left.

subjects moved the handle to the appropriate starting position with the aid of auditory stimuli controlled by the computer. On half of the trials, the starting position was $3^{\circ}$ (of visual angle) to the left of the target; on the other trials, the starting position was $3^{\circ}$ to the right. The computer confirmed that the handle was in the correct position before proceeding with the rest of the trial. The subjects were then required to fixate the target and track it (by eye) as it moved smoothly to the right through a distance of either $4^{\circ}$ or $7^{\circ}$. The velocity of the target followed a sinusoidal time course (a half cycle) with a mean of $5 \% \mathrm{sec}$. The frame also moved smoothly on each trial with a sinusoidal velocity, starting and stopping synchronously with the target. On a randomly determined half of the trials, the frame moved $3^{\circ}$ to the right; on the other trials, it moved $3^{\circ}$ to the left. The display was viewed in complete darkness, and care was taken to ensure that nothing was visible other than the luminous frame and target. To accomplish this, the room lights were automatically turned on between trials to prevent dark adaptation.

The target and frame remained in their final positions for $1 \mathrm{sec}$, and then the display blanked. The subjects were instructed to remain fixated on the location that the target had last occupied. After another $1-\mathrm{sec}$ delay, the computer verified that the handle had not yet moved from the starting position, and an auditory prompt to respond was presented. If the handle did move prematurely, the subjects received the message "HAND MOVED," and the next trial began. Trials with these errors were extremely rare, and any such trials were rerun at the end of the block in which they occurred.

In separate blocks of trials, the subjects performed two different kinds of pointing responses. In one type of block, they were instructed to move the handle so that the thumb pointed to the final location of the target on each trial (location condition). In the other type of block, they were instructed to move the handle to the right through the same distance that the target was perceived to have moved (distance condition). The subjects signaled their responses by pushing a button on the handle. The room lights were then turned on, and after a 2-sec delay the next trial began.

Design. Each subject participated in two sessions on separate days. On Day 1 , the subjects received a practice block consisting of 8 trials, which served to familiarize them with the task and apparatus. Data from that block are not reported. They received six test blocks on Day 1 and eight on Day 2. Each block contained 16 trials: four replications of each combination of target amplitude $\left(4^{\circ}\right.$ or $7^{\circ}$ ) and frame direction (left or right), in a random order. The starting hand position was either $-3^{\circ}$ or $+3^{\circ}$ (randomly selected) equally often for each type of trial. In alternate blocks, the subjects provided either distance or location responses. The response required for the first test block each day (distance or location) was counterbalanced across subjects and days.

\section{Results}

Since the target began its movement from straight ahead $\left(0^{\circ}\right)$, the distance moved by the hand in the distance condition should equal the final location pointed to in the location condition, if distance and location judgments are equally accurate.

Figure 2 shows the mean responses in each condition separately for each combination of target distance and frame direction. First note that there was a significant main effect of target amplitude $[F(1,7)=37.7, p<.001]$. When the target moved $7^{\circ}$, subjects moved their hands farther or pointed farther to the right in the distance and location conditions, respectively, than they did with a $4^{\circ}$ target amplitude. This shows that the subjects were sensitive to the different objective target amplitudes. Next, the direction of frame movement also affected the responses: The subjects pointed farther to the right when the frame moved to the left than when the frame moved to the right $[F(1,7)=19.4, p<.005]$. This shows that the motion of the frame was successful in inducing illusory motion in the target. Finally, there was no overall difference in responses between the distance and location conditions $[F(1,7)=1.8, p<.25]$.

The response condition was involved in several important interactions, as can be seen in Figure 2. First, location responses were more sensitive to the different target amplitudes than were distance responses $[F(1,7)=7.9$, $p<.05$ ]. Next, location and distance responses were affected by the illusion in different ways: The direction of frame movement had a more pronounced effect on responses in the distance condition than in the location condition $[F(1,7)=5.8, p<.05]$.

\section{Discussion}

The important result of Experiment 1 is the interaction between the direction of frame movement and the type of response that subjects produced. The induced motion illusion had a bigger effect when subjects attempted to reproduce the distance that the induction target moved

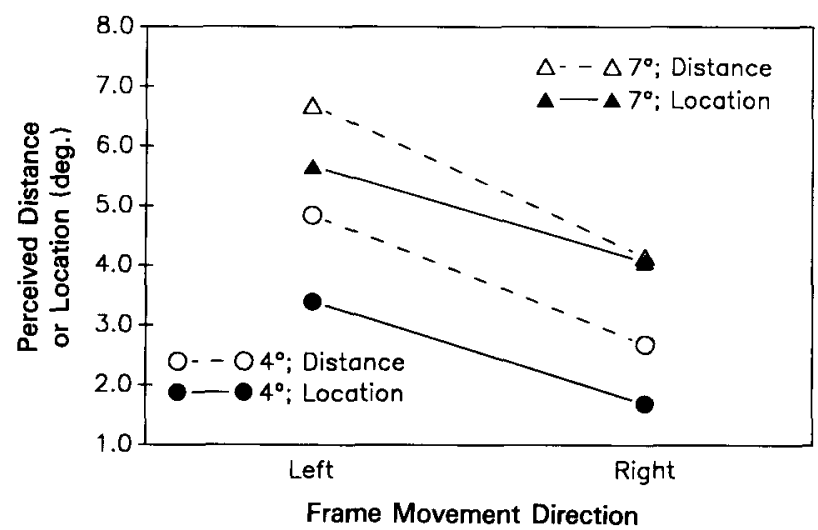

Figure 2. Mean distance moved (distance condition) or final location pointed to (location condition), as a function of the objective target amplitude and direction of frame movement, from Experiment 1 . The initial target position was $0^{\circ}$. 
than when they pointed to the final location of the target. This supports the idea that distance and location information are not derived solely from a common spatial representation, but rather that the two types of judgments appear to tap different internal representations of space. Furthermore, it appears that the two spatial representations are differentially affected by the motion of a surrounding frame.

The present results also bear on an understanding of induced motion. There has been considerable disagreement regarding the precise mechanisms underlying the illusion (see Bacon et al., 1982; Mack, Heuer, Fendrich, et al., 1985; McConkie \& Farber, 1979). Nevertheless, regardless of its source, most earlier theorists implicitly assumed that illusory motion was equivalent to an illusory change in position. One exception is Bacon et al. (1982). They showed that there are some situations in which induced movement of a stationary target will be perceived without a corresponding change in the perceived location of the target. Similarly, the present results suggest that the perception of the distance moved can be different from the perceived change in location. Similar results have been reported by Mack, Heuer, Villardi, and Chambers (1985) for a different visual illusion. Thus, it seems likely that there are two distinct types of spatial information, and that motion of a surrounding frame affects one type more than the other.

\section{EXPERIMENT 2}

An alternate explanation exists for the results of Experiment 1 that would attribute the findings to mechanisms other than those that we have proposed. In Experiment 1, there was a 2 -sec delay between the end of target motion and the prompt for subjects to respond. It is possible that this delay had different effects on spatial memory for distance and spatial memory for location, and some evidence exists suggesting that the two types of information do decay at different rates (Laabs, 1973). Thus, the results may reflect the status of different types of spatial memory, but they may not accurately reflect people's ability to perceive and encode different types of spatial information. To test this possibility, we eliminated the memory component of the task, and repeated Experiment 1.

\section{Method}

Subjects. Twelve Washington University undergraduates participated in two 1-h sessions on separate days. None had served previously. The subjects were paid a base rate of $\$ 3.50$ per session, plus additional bonus payments based on their performance, and were tested individually.

Apparatus, Design, and Procedure. The apparatus, design, and procedure were the same as those in Experiment 1, with only one exception: The subjects were permitted to move the handle to produce their response at any time after the target and frame began to move.

\section{Results}

The results are shown in Figure 3. Target amplitude had a main effect; subjects moved their hand farther or pointed farther to the right with the $7^{\circ}$ target amplitude, in the distance and location conditions, respectively $[F(1,11)=197.6, p<.0001]$. The direction of frame movement also affected the responses, revealing the presence of an induced motion illusion $[F(1,11)=13.3$, $p<.005]$. There was also a difference between distance and location responses overall $[F(1,11)=12.1, p<.01]$.

As in Experiment 1, the important result involves an interaction between the effects of response condition (distance or location) and those of the direction of frame movement. The frame movement direction had a bigger effect on distance responses than on location responses $[F(1,11)=18.6, p<.005]$. No other interactions attained significance.

\section{Discussion}

The present results serve to replicate and extend the findings of Experiment 1. Induced motion illusions have a greater effect on judgments of distance than on judgments of location, even without any contribution of memory decay.

\section{EXPERIMENT 3}

There are two additional alternate explanations for the results of Experiment 1 that would lead to conclusions other than those that we have offered. The first involves the precise locus of induced motion illusions. Bacon et al. (1982) and Mack, Heuer, Fendrich, et al. (1985) have shown that at least some of the illusion can be attributed to a shift in the perceived location of "straight-ahead" caused by the movement of the surrounding frame. Such a straight-ahead shift would cause subjects to misjudge the location of any object that was directly in front of them (Harris, 1974). Since in Experiments 1 and 2 the induction target always started to move from directly in front of the subjects, any response that required an estimation of the initial target location (i.e., distance responses) could theoretically be affected by a straight-ahead shift. Indeed, this hypothesis would predict enhanced illusions in the

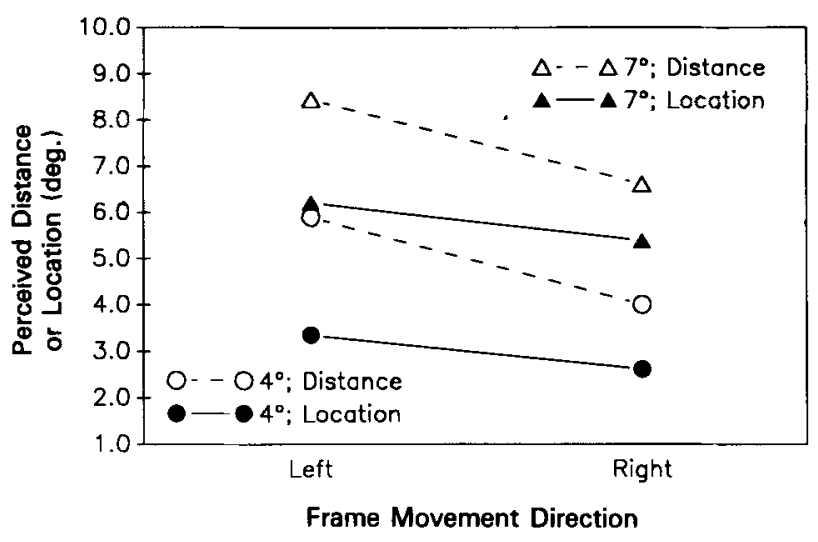

Figure 3. Mean distance moved (distance condition) or final location pointed to (location condition), as a function of the objective target amplitude and direction of frame movement, from Experiment 2. The initial target position was $0^{\circ}$. 
distance condition, where subjects were presumably required to assess the starting location of the target point in addition to its end location, and that was the pattern that was observed. To examine this possibility, subjects in Experiment 3 viewed stimuli similar to those described by Bacon et al. (1982), which can produce an induced motion illusion without a corresponding change in the perceived straight-ahead.

Another reason to question the conclusions of Experiments 1 and 2 involves the behavior of the subjects' eyes during the experiments. Several researchers have shown that people are able to point accurately to the location they are fixating, even in complete darkness (Hill, 1972; Morgan, 1978). If fixation location in the earlier experiments was somehow affected by the response condition, then differences that we observed in the pointing responses may have been mediated by differences in eye position. To examine this possibility, we monitored eye position in Experiment 3.

\section{Method}

Subjects. Eight Washington University undergraduates participated in three 1-h sessions on separate days. None had served previously. The subjects were paid a base rate of $\$ 3.50$ per session, plus additional bonus payments based on their performance, and were tested individually.

Apparatus. The apparatus was the same as that used in Experiment 1 , with the addition of a scleral-reflectance eye-movement monitor (Gulf \& Western, Model 200). The subjects' heads were stabilized by a bite-board.

Procedure. The procedure was the same as that in Experiment 1, with the following exceptions: Instead of being surrounded by a rectangular frame, the induction target appeared superimposed on a background of vertical lines, as shown in Figure 4. Motion was induced in the target by movement of this background. The lines were $1^{\circ}$ apart, contained within an imaginary rectangular frame of exactly the same size and location as the frame used in Experiment $1\left(18^{\circ}\right.$ wide and $10^{\circ}$ high, centered $4.5^{\circ}$ to the right of the target). As in Experiment 1, the target moved smoothly to the right on each trial, through a distance of either $4^{\circ}$ or $7^{\circ}$. The background of lines moved $3^{\circ}$ on each trial, in synchrony with the target, to either the right or the left. When a line reached the edge of the imaginary frame, it disappeared, and a new line appeared at the opposite side of the frame.

Eye-movement evaluation. At the beginning of each trial, the subjects were required to fixate the target, and the computer confirmed that fixation was within $1^{\circ}$ of the nominal target location. If it was not, then the eye-movement monitor was recalibrated, and the trial was rerun from the beginning. Calibration of the monitor consisted of sampling while the subjects fixated each of 11 points spaced evenly across the CRT. Eye position was determined by linear interpolation of the calibration samples.

The target and background began to move 1 sec after the subjects had successfully fixated the target point. The subjects were required to track the target, and eye position was recorded as they did so. If the mean position of the eye was within $2^{\circ}$ of the final target location during the last $100 \mathrm{msec}$ of the pursuit, and if there were no saccades greater than $2^{\circ}$ in amplitude at any time during the pursuit, then the pursuit was accepted. Otherwise, the subjects received an error message, and the trial was rerun at the end of the block. If the pursuit was acceptable, the target remained in its final position for $1 \mathrm{sec}$, the display then blanked, and $1 \mathrm{sec}$ later,

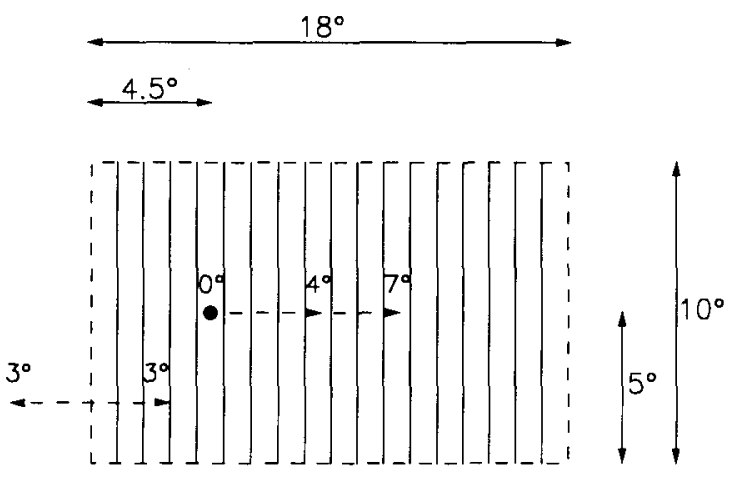

Figure 4. Stimulus display used in Experiment 3. The dashed lines were not visible. On each trial, the target (dot) moved either $4^{\circ}$ or $7^{\circ}$ to the right, and the vertical lines moved $3^{\circ}$ to the right or left. The leading vertical line disappeared when it reached the border of the imaginary frame (dashed lines), and a new line appeared on the opposite side.

the subjects were prompted to produce a manual response, as in Experiment 1.

Design. Each subject participated in three 1-h sessions on separate days. On Day 1, the subjects received six blocks of trials, which served to familiarize them with the task and apparatus: the first two blocks contained 8 trials each, the other blocks contained 16 trials each. The response required (distance or location) alternated from block to block. Data from Day 1 are not reported. On each of the remaining 2 days, the subjects received six blocks of 16 trials each. Each block contained four replications of each combination of target amplitude $\left(4^{\circ}\right.$ or $7^{\circ}$ ) and frame direction (left or right), in a random order. The starting hand position was either $-3^{\circ}$ or $+3^{\circ}$ (randomly selected) equally often for each type of trial. In alternate blocks, the subjects provided either distance or location responses. The response required for the first block each day (distance or location) was counterbalanced across subjects and days.

\section{Results}

Hand movements. The mean responses in the distance and location conditions are shown in Figure 5 separately for each combination of target distance and background direction. The results are very similar to those observed in Experiments 1 and 2. First, the subjects were sensitive to the different objective target amplitudes $[F(1,7)=$ $144.7, p<.001]$. The movement of the background successfully induced illusory motion in the target $[F(1,7)=$ $13.1, p<.01]$. And there was only a small, but reliable, overall difference in distance and location responses $[F(1,7)=6.0, p<.05]$.

As in the earlier experiments, the direction of background movement affected distance responses more than location responses $[F(1,7)=7.8, p<.05]$. Also, location responses were more affected by the target amplitude than were distance responses $[F(1,7)=6.4, p<.05]$.

Eye movements. The mean eye position during the last $100 \mathrm{msec}$ of target motion (shortly before the display blanked) is shown in Table 1 for each condition. As would be expected, the amplitude of target movement affected the final eye position $[F(1,7)=1,272.2, p<.001]$. But 


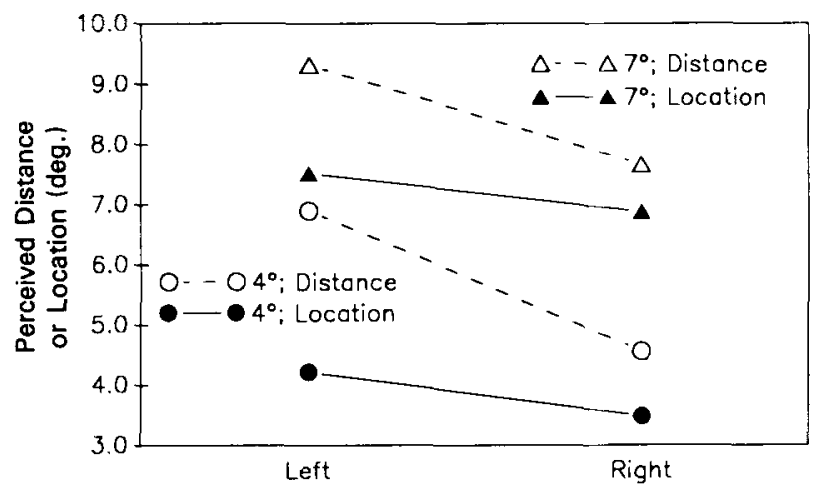

Background Movement Direction

Figure 5. Mean distance moved (distance condition) or final location pointed to (location condition), as a function of the objective target amplitude and direction of background movement, from Experiment 3 . The initial target position was $0^{\circ}$.

eye position did not depend on the response condition or the direction of background movement $[F \mathrm{~s}(1,7)<1]$.

\section{Discussion}

The results of Experiment 3 serve to replicate and extend the findings of Experiments 1 and 2 . As in the earlier experiments, judgments of the distance moved by the induction target were more affected by the motion of the background than were judgments of the target's final position. This result cannot be attributed to differences in eye position or to a straight-ahead shift. Instead, the results suggest that perceived distance moved is not derived from perceived changes in location, but that the two types of information are fundamentally different.

\section{EXPERIMENT 4}

There are some reasons to question the ability of Experiment 3 to rule out possible contributions of changes in the target's perceived starting location. First, we did not directly measure the perceived starting location, and thus it is possible that our display was not successful in eliminating a straight-ahead shift. Second, even in the absence of a straight-ahead shift, it is still possible that the motion of the background distorted subjects' perceptions of the target's initial location. Thus, before concluding that distance and location information are independent, we conducted another experiment, in which we had subjects provide judgments of the location from which the target began its movement (in addition to judgments of distance and final location). This allowed us to measure any distortion in the perceived initial target location, and also to determine the extent to which distorted perceived locations contribute to distorted perceived distances.

\section{Method}

Subjects. Twelve students who were unfamiliar with the present experiments participated in two 1-h sessions on separate days. The subjects were paid a base rate of $\$ 3.50$ per session, plus additional bonus payments based on their performance, and were tested individually.
Apparatus and Procedure. The apparatus was the same as that used in Experiment 3. The procedure was also very similar, with the following exception: In separate blocks of trials, the subjects were asked to provide three different types of judgments about the motion of the target-the distance moved, the final location, and the initial location. The distance and final location conditions were the same as in the previous experiments. In the initial location condition, the subjects were asked to point to the perceived position of the target at the beginning of the trial. All pointing was performed after the target stopped moving.

Design. Each subject participated in two $1-\mathrm{h}$ sessions on separate days. On Day 1, the subjects received practice blocks of 8 trials each in each of the three conditions. Data from those blocks are not reported. They then received two test blocks in each of the three conditions, consisting of 16 trials per block. On Day 2, the subjects served in three blocks of 16 trials each in each of the three conditions. Conditions changed from block to block, and the order of conditions within each cluster of three blocks was counterbalanced.

\section{Results}

The mean distance moved in the distance condition and the mean location pointed to in the location conditions are shown in Table 2 separately for each objective target amplitude and each direction of background motion. There was a significant overall difference between pointing conditions, due primarily to the difference between pointing to the initial and final locations $[F(2,22)=73.1$, $p<.0001]$. There was also a significant effect of target amplitude: The subjects ended with their hands farther to the right in the distance and final location conditions for the $7^{\circ}$ target amplitude than they did for the $4^{\circ}$ amplitude $[F(1,11)=98.5, p<.0001]$. The position pointed to changed very little with target amplitude in the initial location condition, as would be expected, resulting in an interaction between pointing condition and target amplitude $[F(2,22)=52.6, p<.0001]$.

The important results involve the effects of the motion of the background in the various conditions. Overall, subjects responded farther to the right when the background moved to the left, revealing the typical induced motion illusion $[F(1,11)=16.6, p<.005]$. However, the motion of the background had different effects on pointing in the different conditions, resulting in an interaction between background motion and pointing condition $[F(2,22)=$ $23.4, p<.0001]$. As can be seen in Table 2, the illusion magnitude was greater in the distance condition than in the final location condition. Furthermore, the illusion magnitude was small, and it was negative in the initial

Table 1

Mean Final Eye Position (in degrees) in Experiment 3

\begin{tabular}{|c|c|c|c|c|}
\hline & \multicolumn{2}{|c|}{$\begin{array}{l}\text { Objective Target } \\
\text { Amplitude }=4^{\circ}\end{array}$} & \multicolumn{2}{|c|}{$\begin{array}{l}\text { Objective Target } \\
\text { Amplitude }=7^{\circ}\end{array}$} \\
\hline & Distance & Location & Distance & Location \\
\hline Background left & 4.04 & 4.16 & 6.83 & 6.93 \\
\hline Background right & 4.09 & 4.04 & 6.94 & 6.97 \\
\hline Illusion magnitude & -.05 & .12 & -.11 & -.04 \\
\hline
\end{tabular}

Note-The initial target position was $0^{\circ}$ (visual angle). Objective target amplitude is the actual distance moved by the target, as well as the target's final location. Illusion magnitude $=$ background left mean minus background right mean. 
Table 2

Mean Localization Responses (in degrees) from Experiment 4

\begin{tabular}{lcccccccc}
\hline & \multicolumn{2}{c}{ Objective Target Amplitude $=4^{\circ}$} & & \multicolumn{2}{c}{ Objective } & Target Amplitude $=7^{\circ}$ \\
\cline { 2 - 3 } & Distance & $\begin{array}{c}\text { Final } \\
\text { Location }\end{array}$ & $\begin{array}{c}\text { Initial } \\
\text { Location }\end{array}$ & & Distance & Final & Location & $\begin{array}{c}\text { Initial } \\
\text { Location }\end{array}$ \\
\hline Background left & 7.1 & 3.9 & -2.2 & & 9.9 & 7.2 & -2.3 \\
Background right & 5.1 & 3.3 & -1.8 & & 8.4 & 6.6 & -1.9 \\
Illusion magnitude & 2.0 & 0.6 & -0.4 & & 1.5 & & 0.6 & -0.4 \\
\hline
\end{tabular}

Note-Illusion magnitude $=$ background left mean minus background right mean .

location condition. The negative sign of the illusion indicates that the subjects perceived the initial target location to be more to the left when the background moved to the left than when it moved to the right. Thus, motion of the background had opposite effects on the perceived initial and final locations.

In order to compare the magnitude of the illusion in the distance condition with that in the location conditions, we computed a corrected location measure by subtracting each subject's mean response in the initial location condition from that in the final location condition for each objective target amplitude and direction of background motion. Because the former values were of negative sign, this resulted in corrected location scores that were larger than the uncorrected scores. Also, because the motion of the background had opposite effects on perceived initial and final target locations, the correction resulted in enhancing the apparent overall illusion magnitude for the location responses. These corrected location measures are shown plotted in Figure 6. The corrected location measures, along with the original distance responses, were subjected to analysis: Despite the enhanced location illusion, the effect of the background was still greater for distance responses than for the corrected location responses, as can be seen in the figure $[F(1,11)=9.5, p<.05]$.

\section{Discussion}

The results of Experiment 4 provide additional support for the distinction between distance and location information. Motion of the background induced an illusory change in the perceived distance moved by the target that exceeded the sum of the changes induced in the perceived initial and final target locations. This is the same pattern reported by Mack, Heuer, Villardi, and Chambers (1985) for perceptions of Müller-Lyer figures. They found that the distortion of perceived shaft length produced by the wings of the Müller-Lyer figure was greater than the distortion of the end locations of the shaft. Taken together, these results suggest that distance judgments do not simply arise from the comparison of two perceived locations. Rather, spatial information about distance appears to be fundamentally different from information about location.

\section{EXPERIMENT 5}

Moving a limb through a prespecified distance is an uncommon motor behavior-actions are usually directed to specific locations in space (Poulton, 1981). Thus, even if distance and location information are independent, this difference may not have any effect on behavior in most ordinary situations. In our final experiment, we attempted to assess the extent to which differences between distance and location information may have implications for more natural perceptual-motor behaviors such as those that people typically engage in.

Another reason for conducting Experiment 5 involves questions about the aspects of the visual stimuli that the subjects were attending to in Experiments 1-4. If the subjects were attending to different features of the stimulus in the different conditions, then the results may merely reflect differences in people's abilities to encode particular features of a visual stimulus, and not necessarily differences in the type of information available for localization. There is ample evidence that attentional factors can affect visual illusions, and it is important to determine whether such factors contributed to the present findings (Coren \& Porac, 1983; Gogel \& Sharkey, 1988; Kelso, Cook, Olson, \& Epstein, 1975; Tsal, 1984).

To examine these issues, subjects viewed stimuli that were identical to those in Experiment 1. However, in Experiment 5 , the subjects were instructed to point their hands to the final location of the target in all conditions. We varied the position from which the pointing began in a manner similar to that used previously by others, so as to render distance information either more or less reliable (e.g., Honda, 1985; Keele \& Ells, 1972). On some trials, the hand movement began from the same position

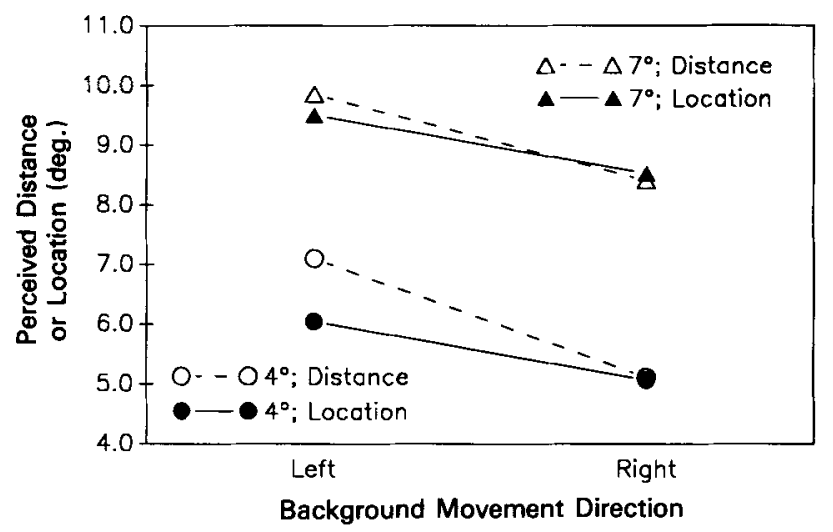

Figure 6. Mean distance responses and corrected location responses from Experiment 4. The corrected location values are differences between perceived final locations and perceived initial locations. See text for further explanation. 
that the target began its movement from. In that situation, subjects could theoretically point to the target either by moving their hands through the perceived distance that the target moved, or by pointing directly to the perceived final location. On other trials, the hand movement began far away from the initial target position. In that case, knowledge of the distance that the target moved would not directly aid pointing to the target's final location. Instead, subjects might be forced to rely on absolute location information. If the type of spatial information that subjects use depends on the position from which the pointing movement begins, then the effect of the induced motion illusion should depend on the starting position of the hand. These manipulations presented subjects with a realistic localization task (that of simply pointing to the position of an object), and also required them, in theory, to extract the same information from the stimulus regardless of where their hands started from (information about the object's final position).

\section{Method}

Subjects. Twelve students who were unfamiliar with the present experiments each participated in two 1-h sessions on separate days. They were paid a base rate of $\$ 3.50$ per session, plus additional bonuses based on their performance, and were tested individually.

Apparatus and Procedure. The apparatus was the same as that used in Experiment 1. The procedure and sequence of events on a trial were also the same, with the following exceptions: Instead of sometimes judging the distance moved by the target, the subjects were instructed to point to the target's final location on every trial. The position from which the hand movement began varied in different blocks of trials. In the home-start condition, the hand always started at the initial (home) position of the target $\left(0^{\circ}\right)$ on each trial throughout a block. In the mixed-start condition, the hand began either $8^{\circ}$ to the left or $12^{\circ}$ to the right of home, equally often throughout a block. These positions were selected because they are at the extremes of the range that we could accurately measure. Since the subjects could not see their hands, they were reminded periodically that all trials in the home-start condition began with the hand at the initial target position. As in the earlier experiments, the subjects could take as much time as they wanted to produce the pointing response.

Design. Each subject served in two sessions on separate days. Day 1 consisted of a practice block with 8 trials followed by six test blocks. On the second day, the subjects completed eight test blocks. Each test block contained 16 trials: four replications of each of the four combinations of target distance $\left(4^{\circ}\right.$ or $\left.7^{\circ}\right)$ and frame direction (left or right). In the mixed-start condition, the hand began equally often from each of the two starting locations $\left(-8^{\circ}\right.$ or $12^{\circ}$ ) on each type of trial. Trials were presented in a random order. In alternate blocks, the subjects performed in either the home-start condition or the mixed-start condition. The condition for the first test block each day was counterbalanced across subjects and days.

\section{Results}

Figure 7 shows the mean location pointed to in each condition, for each combination of target distance and frame direction. There was no overall difference between home-start and mixed-start responses: On the average, pointing did not depend on where the hand started from $[F(1,11)<1]$. The subjects did point consistently farther to the right with larger target amplitudes, as would be expected if they were attending to the task $[F(1,11)=$ $81.3, p<.0001]$. The subjects also pointed farther to the right when the frame moved to the left, indicating the presence of an induced motion illusion $[F(1,11)=24.3$, $p<.001]$.

Most importantly, the effects of the starting location of the hand interacted with those of the direction of frame movement: The effect of the direction of frame movement was larger in the home-start condition than in the mixedstart condition $[F(1,11)=18.8, p<.005]$. No other interactions approached significance.

\section{Discussion}

The results of Experiment 5 show that the position from which a pointing movement begins can have a profound effect on the position pointed to. The illusion induced in the target by movement of the surrounding frame was greater when the subjects pointed to the target starting with their hands at the initial target position, compared with pointing that began with the hand far from the initial target position. This result was obtained even though the subjects had an unlimited amount of time to produce their responses, and the hand starting position was, in principle, not relevant to the task: The subjects were instructed to simply point to the perceived final location of the target on each trial.

Because it was possible for subjects to base their responses directly on information about the distance moved by the target in the home-start condition, but not in the mixed-start condition, the results appear to reflect differential use of information about the perceived distance moved and the perceived final location of the target in the two conditions. When the subjects could rely on distance information, the induced motion illusion had a stronger effect. These findings fit well with those from Experiments 1-4, where it was found that induced motion illusions affected distance judgments more than location judgments.

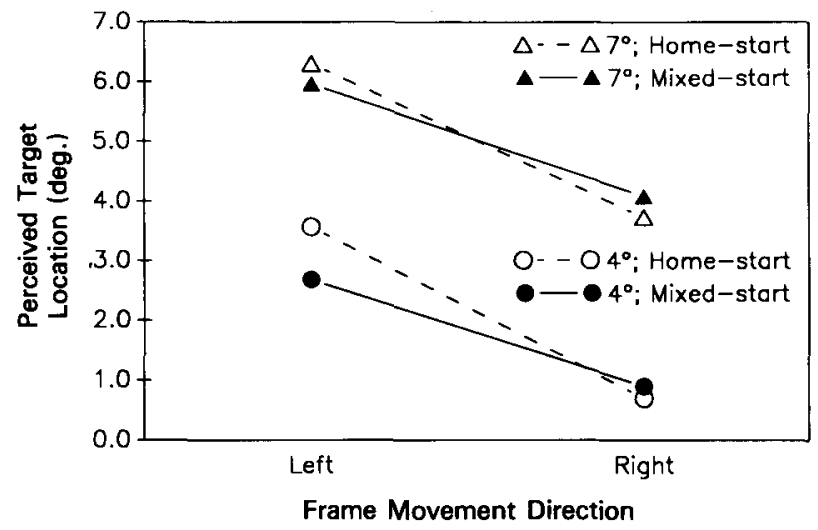

Figure 7. Mean final location pointed to for different hand-starting conditions (Home-start and Mixed-start), as a function of the objective target amplitude and direction of frame movement, from Experiment 5. The initial target position was $0^{\circ}$. 


\section{GENERAL DISCUSSION}

Taken together, the results of the present experiments provide support for the hypothesis that spatial information used for motor localization consists of two different types: information about distance, and information about absolute location. Experiments 1, 2, and 3 demonstrated that the perceived distance moved and the perceived final location of a moving visual target are not always commensurate: Induced motion illusions affected perceived distance more than perceived location. Experiment 4 replicated the earlier experiments, and it also showed that the distortion in perceived distance cannot be explained by a simple combination of distortions in the perceived starting and ending locations of the target. Experiment 5 showed that the distinction between distance and location information can have important implications for pointing movements: Pointing that begins from the initial target position appears to be based on information about the perceived distance of target movement, whereas pointing that begins from elsewhere seems to rely on absolute location information. Thus, people extract more than one type of spatial information from a visual stimulus, and the information used to direct a localization response depends on subtle features of the response.

\section{Perceptions of Space and Time}

The present results have important implications for the nature of a variety of perceptual illusions. Movement of the surrounding frame appears to have induced illusory movement of the target that exceeded the change induced in the target's location. This is consistent with the findings of Bacon et al. (1982) for induced motion illusions, and with those of Mack, Heuer, Villardi, and Chambers (1985) for the Müller-Lyer illusion. A related result was reported by Rosenbaum (1975), who showed that the perceived acceleration of a moving object is not derived from a simple combination of perceived velocity and perceived elapsed time. Apparently, spatial and temporal dimensions that are bound together in the physical world are not necessarily similarly bound in the psychological world.

\section{Nature of Motor Codes}

Our results also bear on the nature of the motor codes that are used to produce goal-directed movements. There has been some controversy regarding how the motor system might specify a given movement. Some evidence exists which suggests that movements can be characterized as arising from a specification of the final desired end location, irrespective of the starting location (see, e.g., Abrams et al., in press; Mays \& Sparks, 1980; Polit \& Bizzi, 1979). However, it also appears that, in some situations, movements may be programmed in terms of the desired magnitude and duration of the force pulse that the muscles apply to the moving member (see, e.g., Abrams et al., 1989, in press; Meyer et al., 1982; Schmidt et al., 1979). The results of the present study suggest that both formulations may be correct: Sometimes movements are programmed in terms of the perceived distance to be moved, whereas at other times, they can be programmed in terms of the final desired location. What is interesting about the present results is the fact that these two alternatives do not always produce identical movements. ${ }^{1}$

\section{Implications for Studies of Visual Localization}

Finally, the present study may help to reconcile conflicting results in prior experiments on localization, and may help to guide future research. In particular, the results may provide some insight into the purported distinction between the nature and quality of spatial information that is available to the perceptual systems and that which is available to the motor systems. As discussed earlier, in a number of different situations, motoric localization responses have been shown to be more accurate than perceptual judgments of the location of a target (e.g., Bridgeman et al., 1979). However, there is also considerable evidence that the motor system is not entirely immune from the effects of factors that influence perceptual judgments (e.g., Abrams et al., in press). As has been seen in the present experiments (e.g., Experiment 5), differential use of distance and location information may underlie differences in localization behavior. This leaves open the possibility that differences observed in prior studies, such as the difference between perceptual and motor responses, may be due at least in part to differential use of the two different types of spatial information in different types of responses. The extent to which these factors may have contributed to earlier results remains a question for further research. Hopefully, the possible existence of two types of spatial information could lead to new insights into the mechanisms underlying spatial localization, and perceptual and motor behavior more generally.

\section{REFERENCES}

Abrams, R. A., Meyer, D. E., \& Kornblum, S. (1989). Speed and accuracy of saccadic eye movements: Characteristics of impulse variability in the oculomotor system. Journal of Experimental Psychology: Human Perception \& Performance, 15, 529-543.

Abrams, R. A., MeYer, D. E., Kornblum, S. (in press). Eyehand coordination: Oculomotor control in rapid aimed limb movements. Journal of Experimental Psychology: Human Perception \& Performance.

Bacon, J. H., Gordon, A., \& Schulman, P. (1982). The effect of two types of induced-motion displays on perceived location of the induced target. Perception \& Psychophysics, 32, 353-359.

Bizzi, E., Polit, A., \& Morasso, P. (1976). Mechanisms underlying achievement of final head position. Journal of Neurophysiology, 39, 435-444.

Bridgeman, B., Kirch, M., \& SPERling, A. (1981). Segregation of cognitive and motor aspects of visual function using induced motion. Perception \& Psychophysics, 29, 336-342.

Bridgeman, B., Lewis, S., Heit, G., \& Nagle, M. (1979). Relation between cognitive and motor-oriented systems of visual position perception. Journal of Experimental Psychology: Human Perception \& Performance, 5, 692-700.

CoRen, S. (1986). An efferent component in the visual perception of direction and extent. Psychological Review, 93, 391-410.

Coren, S., Porac, C. (1983). The creation and reversal of the Mueller-Lyer illusion through attentional manipulation. Perception, 12, 49-54. 
Duncker, K. (1929). Über induzierte Bewegung. Psychologische Forschung, 12, 180-259.

Festinger, L., \& CANON, L. (1965). Information about spatial location based on knowledge about efference. Psychological Review, 72 , 373-384.

FinDLAY, J. M. (1982). Global visual processing for saccadic eye movements. Vision Research, 22, 1044-1045.

GHEz, C. (1979). Contribution of central programs to rapid limb movement in the cat. In H. Asanuma \& V. J. Wilson (Eds.), Integration in the nervous system. Tokyo: Igaku-Shoin.

Gogel, W. C., Sharkey, T. J. (1988, November). Measuring attention using induced motion. Paper presented at the meeting of the Psychonomic Society, Chicago, IL.

Hallett, P. E., \& Lightstone, A. D. (1976). Saccadic eye movements to flashed targets. Vision Research, 16, 107-114.

Hallett, M., Shahani, B. T., Young, R. R. (1975). EMG analysis of stereotyped voluntary movements in man. Journal of Neurology, Neurosurgery, \& Psychiatry, 38, 1154-1162.

Hansen, R. M. (1979). Spatial localization during pursuit eye movements. Vision Research, 19, 1213-1221.

Hansen, R. M., Skavenski, A. A. (1977). Accuracy of eye position information for motor control. Vision Research, 17, 919-926.

Hansen, R. M., \& Skavenski, A. A. (1985). Accuracy of spatial localizations near the time of saccadic eye movements. Vision Research, $25,1077-1082$.

Harris, C. S. (1974). Beware of the straight-ahead shift-a nonperceptual change in experiments on adaptation to displaced vision. Perception, 3, 461-476.

HeLmHoltz, H. voN (1963). A treatise on physiological optics (J. P. C. Southall, Ed. and Trans.). New York: Dover. (Original work published 1866)

Hill, A. L. (1972). Direction constancy. Perception \& Psychophysics, $11,175-178$.

Honda, H. (1984). Eye-position signals in successive saccades. Perception \& Psychophysics, 36, 15-20.

HondA, H. (1985). Spatial localization in saccade and pursuit-eyemovement conditions: A comparison of perceptual and motor measures. Perception \& Pychophysics, 38, 41-46.

Keele, S. W., E Ells, J. G. (1972). Memory characteristics of kinesthetic information. Journal of Motor Behavior, 4, 127-134.

KeLSo, J. A. S. (1977). Motor control mechanisms underlying human movement reproduction. Joumal of Experimental Psychology: Human Perception \& Performance, 3, 529-543.

Kelso, J. A. S., Cook, E., Olson, M. E., \& Epstein, W. (1975). Allocation of attention and the locus of adaptation to displaced vision. Joumal of Experimental Psychology: Human Perception \& Performance, 1, 237-245.

LAABS, G. J. (1973). Retention characteristics of different reproduction cues in motor short-term memory. Journal of Experimental Psychology, 100, 168-177.

LACKNER, J. R., LeVINE, M. S. (1981). The guidance of saccadic eye movements to perceptually mislocalized visual and non-visual targets. Aviation, Space, \& Environmental Medicine, 52, 461-465.

Mack, A., Heuer, F., Fendrich, R., Villardi, K., \& Chambers, D. (1985). Induced motion and oculomotor capture. Journal of Experimental Psychology: Human Perception \& Performance, 11, 329-345.

Mack, A., Heuer, F., Villardi, K., \& Chambers, D. (1985). The dissociation of position and extent in Müller-Lyer figures. Perception \& Psychophysics, 37, 335-344.

MacNeilage, P. F. (1970). Motor control of serial ordering of speech. Psychological Review, 77, 182-196.

MarteniuK, R. G. , Roy, E. A. (1972). The codability of kinesthetic location and distance information. Acta Psychologica, 36, 471-479.

Matin, L. (1972). Eye movements and perceived visual direction. In
D. Jameson \& L. Hurvich (Eds.), Handbook of sensory physiology: Vol. 7. Pt. 4. Visual psychophysics (pp. 331-380). Berlin: Springer.

Matin, L., Matin, E., \& Pearce, D. G. (1969). Visual perception of direction when voluntary saccades occur: I. Relation of visual direction of a fixation target extinguished before a saccade to a flash presented during the saccade. Perception \& Pychophysics, 5, 65-80.

Matin, L., Picoult, E., Stevens, J. K., Edwards, M. W., Young, D., MACARThUR, R. (1982). Oculoparalytic illusion: Visual-field dependent spatial mislocalizations by humans partially paralyzed with curare. Science, 216, 198-201.

MAYS, L. E., SPARKs, D. L. (1980). Saccades are spatially, not retinotopically coded. Science, 208, 1163-1164.

MCCONKIE, A. B., \& FARBER, J. M. (1979). Relation between perceived depth and perceived motion in uniform flow fields. Journal of Experimental Psychology: Human Perception \& Performance, 5, 501-508.

Meyer, D. E., Abrams, R. A., Kornhlum, S., Wright, C. E., \& SMITH, J. E. K. (1988). Optimality in human motor performance: Ideal control of rapid aimed movements. Psychological Review, 95 , 340-370.

Meyer, D. E., Smith, J. E. K., \& Wright, C. E. (1982). Models for the speed and accuracy of aimed movements. Psychological Review, 89, 449-482.

Miller, J. M. (1980). Information used by the perceptual and oculomotor systems regarding the amplitude of saccadic and pursuit eye movements. Vision Research, 20, 59-68.

Morgan, C. L. (1978). Constancy of egocentric visual direction. Perception \& Psychophysics, 23, 61-68.

Polit, A., \& BizzI, E. (1979). Characteristics of motor programs underlying arm movements in monkeys. Joumal of Neurophysiology, 42, 183-194.

Poulton, E. C. (1981). Human manual control. In V. Brooks (Ed.), Handbook of physiology: The nervous system: Vol. 2. Motor control (pp. 1337-1389). Baltimore: American Physiological Society.

RoBinson, D. A. (1964). The mechanics of human saccadic eye movement. Journal of Physiology, 174, 245-264.

Rosenbaum, D. A. (1975). Perception and extrapolation of velocity and acceleration. Joumal of Experimental Psychology: Human Perception \& Performance, 1, 395-403.

SAKITT, B. (1980). A spring model and equivalent neural network for arm posture control. Biological Cybernetics, 37, 227-234.

Schmidt, R. A., Zelaznik, H., Hawkins, B., Frank, J. S., \& QuinN, J. T., JR. (1979). Motor-output variability: A theory for the accuracy of rapid motor acts. Psychological Review, 86, 415-451.

Stelmach, G. E., Kelso, J. A. S., Wallace, S. A. (1975). Preselection in short-term motor memory. Journal of Experimental Psychology: Human Learning \& Memory, 1, 745-755.

TSAL, Y. (1984). A Mueller-Lyer illusion induced by selective attention. Quarterly Journal of Experimental Psychology, 36A, 319-333.

WoNG, E., MACK, A. (1981). Saccadic programming and perceived location. Acta Psychologica, 48, 123-131.

\section{NOTE}

1. In a study involving rapid aimed hand movements, Abrams et al. (in press) concluded that a single movement may be programmed in terms of both distance and location. They found that the initial ballistic impulse of a rapid movement appeared to be programmed in terms of the perceived distance between the starting and target locations, whereas the final homing in on the target was governed by the perceived absolute location.

(Manuscript received December 2, 1988; revision accepted for publication October $13,1989$. 